\title{
Nonlocal solutions of parabolic equations with strongly elliptic differential operators
}

\author{
Irene Benedetti \\ Department of Mathematics and Computer Sciences, University of Perugia \\ I-06123 Italy, e-mail: irene.benedetti@dmi.unipg.it \\ Luisa Malaguti \\ Department of Sciences and Methods for Engineering, University of Modena and Reggio Emilia \\ I-42122 Italy, e-mail: luisa.malaguti@unimore.it \\ Valentina Taddei \\ Department of Sciences and Methods for Engineering, University of Modena and Reggio Emilia \\ I-42122 Italy, e-mail: valentina.taddei@unimore.it
}

\begin{abstract}
The paper deals with second order parabolic equations on bounded domains with Dirichlet conditions in arbitrary Euclidean spaces. Their interest comes from being models for describing reaction-diffusion processes in several frameworks. A linear diffusion term in divergence form is included which generates a strongly elliptic differential operator. A further linear part, of integral type, is present which accounts of nonlocal diffusion behaviours. The main result provides a unifying method for studying the existence and localization of solutions satisfying nonlocal associated boundary conditions. The Cauchy multipoint and the mean value conditions are included in this investigation. The problem is transformed into its abstract setting and the proofs are based on the homotopic invariance of the Leray-Schauder topological degree. A bounding function (i.e. Lyapunov-like function) theory is developed, which is new in this infinite dimensional context. It allows that the associated vector fields have no fixed points on the boundary of their domains and then it makes possible the use of a degree argument.
\end{abstract}

AMS Subject Classification: Primary 35K20. Secondary 34B10, 47H11, 93D30.

Keywords: Parabolic equations; multipoint and mean value conditions; degree theory; Lyapunov-like functions.

\section{Introduction}

The paper deals with the second order parabolic equation

$$
\frac{\partial u(t, \xi)}{\partial t}=\sum_{i, j=1}^{n} \frac{\partial}{\partial \xi_{i}}\left(a_{i, j}(\xi) \frac{\partial u(t, \xi)}{\partial \xi_{j}}\right)+\int_{D} k(\xi, y) u(t, y) d y-b u(t, \xi)+g(t, u(t, \xi))
$$

with $t \in[0, T]$ and $\xi \in D \subset \mathbb{R}^{n}$, where $D$ is a bounded domain with a sufficiently regular boundary $\partial D$. The coefficients $a_{i, j} \in C^{1}(\bar{D})$ for $i, j=1, \ldots, n$, are symmetric i.e.

$$
a_{i, j}(\xi)=a_{j, i}(\xi), \xi \in \bar{D} \quad \text { for } i, j=1, \ldots, n \text { with } i \neq j
$$


and there is a value $C_{0}>0$ such that

$$
C_{0}\|\sigma\|^{2} \leq \sum_{i, j=1}^{n} a_{i, j}(\xi) \sigma_{i} \sigma_{j} \text { for all } \sigma \in \mathbb{R}^{n} .
$$

Moreover $b>0$ is a prescribed constant, $k: D \times D \rightarrow \mathbb{R}$ and $g:[0, T] \times \mathbb{R} \rightarrow \mathbb{R}$ are two given maps. The solution is subject to the Dirichlet boundary conditions

$$
u(t, \xi)=0, \quad \text { for } t \in[0, T], \xi \in \partial D .
$$

Equation (1.1) is a model for reaction-diffusion processes in many frameworks and hence it is widely investigated. We refer to the recent monographs [11, [15], 27] and [35] for a wide discussion on parabolic dynamics. The symmetric second order differential operator in its r.h.s. accounts of diffusion behaviours of a punctual type while the nonlocal term in integral form includes long distance diffusive interactions or memory effects. When $a_{i, j}(\xi) \equiv$ $\delta_{i, j}=\left\{\begin{array}{ll}0 & i \neq j \\ 1 & i=j\end{array}\right.$, the differential term on the right hand side of (1.1) simply reduces to the Laplace operator and hence (1.1) becomes

$$
u_{t}(t, \xi)=\Delta u(t, \xi)+\int_{D} k(\xi, y) u(t, y) d y-b u(t, \xi)+g(t, u(t, \xi)), \quad t \in[0, T], \xi \in D .
$$

We always assume that

(i) $g$ is continuous and there exist $L>0$ and $\beta \in(0,1)$ such that

$$
|g(t, \xi)-g(t, y)| \leq L \max \left\{|\xi-y|^{\beta},|\xi-y|\right\}, \text { for } t \in[0, T], \xi, y \in \mathbb{R}
$$

(ii) $k \in L^{\infty}(D \times D)$ and $0 \leq k(\xi, y) \leq 1$ for a.a. $\xi, y \in D$.

By the estimate in (1.5) (i) the function $g$ has a sublinear growth in its second variable $\xi$ when $|\xi| \rightarrow \infty$, for every $t \in[0, T] ; g$ is also Hölder continuous with exponent $\beta$ in $\xi$ for every $t$ and then, in particular, $g(t, \xi)$ may approach $g(t, 0)$ as $|\xi|^{\beta}$ when $\xi \rightarrow 0$.

When $k(\xi, y):=h(\xi-y)$ for a.a. $\xi, y \in D$ with $h \in L^{\infty}(D)$ and $0 \leq h(\xi) \leq 1$ for a.a. $\xi \in D$, the integral term in (1.1) can be written in the form $h * u(t, \cdot)$, i.e. is a convolution product with convolution kernel $h$.

As usual $L^{p}(D)$ denotes the Lebesgue space $L^{p}(D, \mathbb{R})$ and we always restrict to the case when

$$
1<p<\infty .
$$

Under conditions (1.2) and (1.3) the linear elliptic partial differential operator in divergence form $A_{p}: W^{2, p}(D) \cap W_{0}^{1, p}(D) \rightarrow L^{p}(D)$ given by

$$
A_{p}(v)(x)=\sum_{i, j=1}^{n} \frac{\partial}{\partial x_{i}}\left(a_{i j}(x) \frac{\partial v(x)}{\partial x_{j}}\right)
$$

is well-defined and it is the infinitesimal generator of an analytic semigroup of contractions $\{S(t)\}_{t \geq 0}$ in $L^{p}(D)$ (see e.g. [29, Theorem 3.6 p. 215]); we refer to Section[2 for an additional discussion about this semigroup. 
The abstract formulation of (1.1) takes the form

$$
x^{\prime}(t)=A x(t)+f(t, x(t)), \quad t \in[0, T], x \in L^{p}(D)
$$

with

$$
x(t):=u(t, \cdot), \quad \text { for } t \in[0, T] .
$$

As usual (1.7) is obtained by equation (1.1) when $u$ is no longer considered as a function of the variables $t$ and $\xi$, but as a mapping $t \longmapsto u(t, \cdot)$ with $t \in[0, T]$ and $u(t, \cdot)$ in a suitable function space. For short in (1.7) we simply denote with $A$ the linear operator $A_{p}$ while the function $f:[0, T] \times L^{p}(D) \rightarrow L^{p}(D)$ includes all the additional terms in (1.1) (see formula (4.3) in Section (4).

By a solution of (1.1) we mean a function $u:[0, T] \times D \rightarrow \mathbb{R}$ with $u(t, \cdot) \in L^{p}(D)$ for all $t \in$ $[0, T]$ such that the corresponding function $x$ introduced in (1.8) belongs to $C\left([0, T], L^{p}(D)\right)$ and it is a solution of (1.7) in integral form, i.e. $x$ is a mild solution (see Definition (3.1)) of (1.7).

The existence of solutions to (1.1) which satisfy given nonlocal conditions displays a growing interest for the possibility of these trajectories to capture additional information about the dynamics. We mention, for instance, the mean value condition.

$$
u(0, \xi)=\frac{1}{T} \int_{0}^{T} u(t, \xi) d t, \quad \text { for a.a. } \xi \in D
$$

and the multipoint condition

$$
u(0, \xi)=\sum_{i=1}^{q} \alpha_{i} u\left(t_{i}, \xi\right) \text { with } t_{i} \in(0, T], \alpha_{i} \in \mathbb{R}, i=1, \ldots, q \quad \text { and a.a. } \xi \in D .
$$

The linear parabolic case with no integral term and boundary conditions as in (1.10) is in Chabrowski [7]; the study is based on a maximum principle and the use of a Green function. The model in Deng [10] deals with the evolution of a small quantity of gas in a tube; the nonlocal condition is of integral type (see (1.9) ) and $t$ varies on a half-line; the nonlinear term is smooth and also the asymptotic behaviour of the solution at infinity is discussed. The nonlocal condition in Jackson [18] is quite general and possibly nonlinear. Pao [28] treated the existence and multiplicity of solutions between a pair of ordered upper and lower solution again in a smooth model which also includes a nonlocal initial condition. Infante-Maciejewski [17] and Xue [34] studied systems of two equations with an elliptic part given by the Laplace operator; while the latter is based on a fixed point argument, in the former a degree argument is used and the appearance of positive solutions is proved; strong growth restrictions on the terms are assumed in both papers. The model introduced by Zhu-Li [37] is quite general, again with integral nonlocal conditions, but the growth and regularity conditions are rather strong and given in implicit form. A degree argument is used also by Benedetti-Loi-Taddei 3, combined with an approximation solvability method and it seems especially useful for treating the case when the nonlinearity depends on some weighted mean value of the solution. At last the model proposed by Viorel [31] has an autonomous nonlinearity of polynomial type with a superlinear growth at infinity.

We will prove the following result on the existence of solutions satisfying the above boundary conditions. Notice that our model has quite general regularity conditions and no 
growth restrictions on its term. As usual the symbol $|D|$ denotes the Lebesgue measure of the set $D$.

Theorem 1.1. Consider equation (1.1) with $a_{i, j} \in C^{1}(\bar{D}), i, j=1, \ldots, n$ satisfying (1.2), (1.3). Assume conditions in (1.5) and let $b>L+|D|$. Then problem (1.1)-(1.4)

(i) admits a solution satisfying condition (1.9);

(ii) admits a solution satisfying condition (1.10) provided that

$$
\sum_{i=1}^{q}\left|\alpha_{i}\right| \leq 1 .
$$

The paper contains a wider discussion which involves the quite general nonlocal condition

$$
x(0)=M(x)
$$

where $M: C\left([0, T], L^{p}(D)\right) \rightarrow L^{p}(D)$ and $x$ is the function defined in (1.8). It is clear that (1.9), (1.10) and the Cauchy condition $x(0)=x_{0}=u(0, \cdot)$ satisfy (1.11). Notice moreover that

(i) the periodic condition: $M(x)=x(T)=u(T, \cdot)$;

(ii) the antiperiodic condition: $M(x)=-x(T)=u(T, \cdot)$

are special cases of (1.10). We remark that (1.11) also includes nonlinear conditions such as

$$
u(0, \xi)=G\left(\int_{0}^{T} h(t) u(t, \xi) d t\right), \quad \xi \in D
$$

with suitable $h:[0, T] \rightarrow \mathbb{R}$ and $G: \mathbb{R} \rightarrow \mathbb{R}$ introduced for instance in 4] (see Example 5) in the framework of age-population models.

The proof of Theorem 1.1 is in Section 4 where there is a quite general discussion about problem(1.1)-(1.4)-(1.11) (see Theorem 4.1). The results are based on a unifying approach of topological type on the abstract setting, i.e. for equation (3.1) (see Section 3); a degree argument, in particular, is used there which makes then possible to avoid strong restrictions on the terms of (1.1) as already noted about Theorem 1.1. On the other hand, the involved vector fields need to be fixed-points free on their boundary; the property is obtained by a bounding function (i.e. Lyapunov-like) method which is original in this infinite dimensional setting; the method is discussed in Section 5. Section 2 contains some notation and preliminary results.

Several Banach spaces appear in this paper; we simply use the symbol $\|\cdot\|$ to denote the norm in all of them when it is clear from the context which is, each time, the involved space. The symbol $E^{*}$ stands for the dual space of $E$. 


\section{Preliminary results and notation}

Let $E$ be a Banach space. A family of linear, bounded operators $S(t): E \rightarrow E$, for $t$ in the interval $[0, \infty)$, is called a $C_{0}$-semigroup if the following conditions are satisfied:

(a) $S(0)=I$;

(b) $S(t+r)=S(t) S(r)=S(r) S(t)$ for $t, r \in[0, \infty)$;

(c) the function $t \rightarrow S(t) x$ is continuous on $[0, \infty)$, for every $x \in E$.

The infinitesimal generator of $S(t)$ is the linear operator $A$ defined by

$$
A x=\lim _{h \rightarrow 0^{+}} \frac{(S(h)-I) x}{h}, x \in D(A)
$$

with

$$
D(A):=\left\{x \in E: \lim _{h \rightarrow 0^{+}} \frac{(S(h)-I) x}{h} \text { exists }\right\} .
$$

We refer to [25], 29], 33] for the theory of semigroups. Here we only restrict to those properties which are needed in our investigation.

As a straightforward consequence of (c) (see e.g. [29, Theorem 2.4 p.4]), we obtain

$$
\lim _{h \rightarrow 0} \frac{1}{h} \int_{t}^{t+h} S(s) x d s=S(t) x, t \geq 0, x \in E .
$$

Every $C_{0}$-semigroup is bounded, for $t$ in a bounded interval, (see e.g. [29, Theorem 2.2 p.4]), in the space $\mathcal{L}(E)$ of linear, bounded operators. When further $\|S(t)\| \leq 1$ for $t \geq 0,\{S(t)\}_{t \geq 0}$ is said to be a contraction semigroup. It is easy to see that every contraction semigroup satisfies

$$
S(t) r \bar{B} \subseteq r \bar{B}, \quad \text { for } r>0, t \geq 0,
$$

where $B$ is the open unit ball in $E$ centered at 0 . The semigroup $\{S(t)\}_{t \geq 0}$ is said:

compact if $S(t)$ is compact, for $t>0$;

uniformly differentiable if the map $t \longmapsto S(t)$ defined on $[0, \infty)$ with values in $\mathcal{L}(E)$ is differentiable for every $t>0$ (see e.g. [33, Definition 6.3.2]).

For $0<\theta \leq \pi$ define the sector

$$
C_{\theta}:=\{z \in \mathbb{C}:-\theta<\arg z<\theta\} .
$$

Clearly

$$
\bar{C}_{\theta}=\{z \in \mathbb{C}:-\theta \leq \arg z \leq \theta\} \cup\{0\} .
$$

Definition 2.1. ([33, Definition 7.1.1]) The $C_{0}$-semigroup $\{S(t)\}_{t \geq 0}$ is said to be analytic if there is $\theta \in(0, \pi]$ and a mapping $\tilde{S}: \bar{C}_{\theta} \rightarrow \mathcal{L}(E)$ such that

(i) $S(t)=\tilde{S}(t), t \geq 0$;

(ii) $\tilde{S}(z+w)=\tilde{S}(z) \tilde{S}(w)$ for $z, w \in \bar{C}_{\theta}$; 
(iii) $\lim _{z \in \bar{C}_{\theta}, z \rightarrow 0} \tilde{S}(z) x=x$ for $x \in E$;

(iv) the mapping $z \longmapsto \tilde{S}(z)$ is analytic from $\bar{C}_{\theta}$ to $\mathcal{L}(E)$.

Lemma 2.1. ([33, Corollary 3.5.1] Let $A: D(A) \subseteq E \rightarrow E$ be the infinitesimal generator of a $C_{0}$-semigroup. The map $\|\cdot\|_{D(A)}: D(A) \rightarrow \mathbb{R}$ given by

$$
\|x\|_{D(A)}:=\|x\|+\|A x\|
$$

defines a norm in $D(A)$, called graph norm, with respect to which $D(A)$ is a Banach space.

We make use, in the sequel, of the following compactness condition which involves the graph norm.

Proposition 2.1. [33, Corollary 6.3.2] Let $A: D(A) \subseteq E \rightarrow E$ be the infinitesimal generator of a uniformly differentiable $C_{0}$-semigroup of contractions. If $D(A)$, endowed with the graph norm, is compactly embedded in $E$, then the semigroup generated by $A$ is compact.

We complete this brief discussion about semigroup theory with an important result about the semigroup generated by the elliptic operator introduced in (1.6).

Theorem 2.1. Consider the linear operator $A_{p}$ defined in (1.6). When conditions (1.2), (1.3) are satisfied, the semigroup generated by $A_{p}$ is compact.

Proof. The linear operator $A_{p}$ is the infinitesimal generator of an analytic semigroup of contractions on $L^{p}(D)$ (see e.g. [29, Theorem 3.6 p.215 ]). In particular, the semigroup is uniformly differentiable. $A_{p}$ is also strongly elliptic of order 2 by conditions (1.2), (1.3). Therefore, the following estimate holds (see e.g [29, Theorem 3.1 p. 212])

$$
\|v\|_{W^{2, p}(D)} \leq C\left(\|A v\|_{L^{p}(D)}+\|v\|_{L^{p}(D)}\right)=C v \|_{D\left(A_{p}\right)}, \quad v \in D\left(A_{p}\right),
$$

for some $C>0$. Hence, the Banach space $\left(D\left(A_{p}\right),\|\cdot\|_{D\left(A_{p}\right)}\right)$ is continuously embedded into the space $\left(D\left(A_{p}\right),\|\cdot\|_{W^{2, p}(D)}\right)$. By Sobolev-Rellich-Kondrachov Theroem (see e.g. 33 , Theorem 1.5.4] $)$ the embedding of $\left(D\left(A_{p}\right),\|\cdot\|_{W^{2, p}(D)}\right)$ into $\left(D\left(A_{p}\right),\|\cdot\|_{L^{p}(D)}\right)$ is compact. We obtained that the embedding of $\left(D\left(A_{p}\right),\|\cdot\|_{D\left(A_{p}\right)}\right)$ into $\left(D\left(A_{p}\right),\|\cdot\|_{L^{p}(D)}\right)$ is compact. We complete the proof by means of Proposition 2.1 .

Definition 2.2. ([19, Definition 4.2.1]) A sequence $\left\{f_{n}\right\} \subset L^{1}([a, b], E)$ is said to be semicompact if it is integrably bounded, i.e. $\left\|f_{n}(t)\right\| \leq \nu(t)$ for a.a. $t \in[a, b]$ and all $n \in \mathbb{N}$ with $\nu \in L^{1}([a, b], E)$, and the set $\left\{f_{n}(t)\right\}$ is relatively compact for a.a. $t \in[a, b]$.

We recall now a useful compactness result in the space of continuous function, involving semicompact sequences.

Theorem 2.2. ([19, Theorem 5.1.1.]) Let $G: L^{1}([a, b], E) \rightarrow C([a, b], E)$ be an operator satisfying the following conditions

(i) there exists $\sigma \geq 0$ such that

$$
\|G f-G g\| \leq \sigma\|f-g\|
$$


(ii) for any compact $K \subset E$ and sequence $\left\{f_{n}\right\} \subset L^{1}([a, b], E)$ such that $\left\{f_{n}(t)\right\} \subset K$ for a.a. $t \in[a, b]$, the weak convergence $f_{n} \rightarrow f_{0}$ in $L^{1}([a, b], E)$ implies that $G f_{n} \rightarrow G f_{0}$.

Then, for every semicompact sequence $\left\{f_{n}\right\} \subset L^{1}([a, b], E)$ the sequence $\left\{G f_{n}\right\}$ is relatively compact in $C([a, b], E)$ and, moreover, if $f_{n} \rightarrow f_{0}$ then $G f_{n} \rightarrow G f_{0}$.

Example 2.1. Let $\{S(t)\}_{t \geq 0}$ be a (not necessarily compact) $C_{0}$-semigroup. Then, the associated Cauchy operator $G: L^{1}([a, b], E) \rightarrow C([a, b], E)$ defined by

$$
G f(t)=\int_{a}^{t} S(t-s) f(s) d s
$$

satisfies conditions (i) and (ii) in Theorem [2.2 (see e.g. [19, Lemma 4.2.1]).

A function $f: X \rightarrow Y$ between the Banach spaces $X$ and $Y$ is said to be completely continuous if it is continuous and maps bounded subsets $U \subset X$ into relatively compact subsets of $Y$. Given a nonempty, open and bounded set $U \subset X$ and a completely continuous map $g: \bar{U} \rightarrow X$ satisfying $x \neq g(x)$ for all $x \in \partial U$, then for the corresponding vector field $i-g$ (where $i$ denotes the identity map on $X$ ) the Leray-Schauder topological degree $\operatorname{deg}(i-g, \bar{U})$ is well-defined (see, e.g. [22, 23]) and it satisfies the usual properties.

\section{Nonlocal solutions in Banach spaces}

In this part we deal with the equation (1.7). Indeed, in order to lead a discussion as general as possible, we let $t$ varying in an arbitrary interval $[a, b]$ and $x$ in a reflexive Banach space $E$ i.e. we consider

$$
x^{\prime}(t)=A x(t)+f(t, x(t)), \quad t \in[a, b], x \in E .
$$

We assume that

(A) $A$ is a linear, not necessarily bounded, operator with $A: D(A) \subset E \rightarrow E$ and it generates a compact $C_{0}$-semigroup of contractions $S:[0, \infty) \rightarrow \mathcal{L}(E)$ (see Section 2 for details);

(f) the function $f:[a, b] \times E \rightarrow E$ is continuous and for every $\Omega \subset E$ bounded there is $\nu_{\Omega} \in L^{1}([a, b])$ such that $\|f(t, x)\| \leq \nu_{\Omega}(t)$ for a.a. $t \in[a, b]$ and $x \in \Omega$.

We consider mild solutions of (3.1), that is functions $x \in C([a, b], E)$ which satisfy (3.1) in integral form; more precisely

Definition 3.1. A function $x \in C([a, b], E)$ is said to be a mild solution of the equation (3.1) if

$$
x(t)=S(t-a) x(a)+\int_{a}^{t} S(t-s) f(s, x(s)) d s, \quad t \in[a, b] .
$$

We combine equation (3.1) with a nonlocal condition

$$
x(a)=M(x),
$$

where $M: C([a, b], E) \rightarrow E$. We assume that (see below formula (2.2) for the symbol $B$ ) 
$\left(m_{0}\right)$ there exists a positive constant $r$ such that $M(C([a, b], r \bar{B})) \subseteq r \bar{B}$;

$\left(m_{1}\right)$ if $\left\{x_{n}\right\} \subset C([a, b], r \bar{B})$ and $x_{n}(t) \rightarrow x(t)$ for $t \in(a, b]$ with $x \in C([a, b], E)$, then $M\left(x_{n}\right) \rightarrow M(x)$ as $n \rightarrow \infty$.

Remark 3.1. By condition $\left(m_{1}\right)$, the function $M$ is clearly continuous, when restricted to $C([a, b], r \bar{B})$.

The study of problem (3.1)-(3.3) very naturally leads to be performed with a topological method. It was initiated by Byszewski [6] and the nonlocal condition there is of the type

$$
x(a)+g\left(t_{1}, \ldots, t_{p}, x\left(t_{1}\right), \ldots, x\left(t_{p}\right)\right)=x_{0}, \quad a<t_{1}<\ldots<t_{p} \leq b,
$$

hence possibly nonlinear. Additional results can be found in [3], [5], 8], 20], 24], 34] and [38] (see also the references there). A fixed point theorem is used in all these papers such as the Banach contraction principle, the Schauder fixed point theorem or the fixed point theorem for condensing maps; hence, strong growth and regularity assumptions are needed, such as the compactness of the function involved in the nonlocal condition or the sublinearity of the nonlinear term with respect to the variable $x$.

A topological degree was introduced by Ćwiszewski-Kokocki [9] (see also 21]) for the study of a periodic problem. A new approximation solvability method, involving a degree argument, was used in Benedetti-Loi-Taddei [3], it allows to treat nonlinear terms satisfying the very general growth condition in $(f)$ but requires the continuity of $f(t, \cdot)$ with respect to the weak topology in $E$ for a.a. $t$ and the linearity of $M$. Despite, like the above mentioned results, in this paper we use the invariance of an appropriate topological degree by an homotopic field, our new technique allows to assume the very general growth condition $(f)$ and we do not need any compactness or linearity conditions on $M$. However, in order that such an invariance is satisfied, the vector fields need to be fixed-points free on the boundary of their domains (see Section (2). This is usually known as the transversality condition which is strictly related to the notion of bounding function (i.e. Lyapunov-like function, see Definition (3.2) and Section 5).

In some cases (see e.g. [2], 8], [20] and [34]) the discussion took place in the multivalued setting. We claim that, with minor changes, the present investigation can be generalized to multivalued dynamics.

Definition 3.2. Let $K \subset E$ be nonempty, open and bounded. A function $V: E \rightarrow \mathbb{R}$ satisfying

(V1) $V / \partial K=0, \quad V / K \leq 0$

(V2) $\liminf _{h \rightarrow 0^{-}} \frac{V(x+h f(t, x))}{h}<0$, for all $t \in(a, b]$ and $x \in \partial K$;

is said to be a bounding function for equation (3.1).

By means of this tool we can use the Leray-Schauder degree theory in order to prove the following result. 
Theorem 3.1. Consider the b.v.p. (3.1)-(3.3) under conditions (A), (f), $\left(\mathrm{m}_{0}\right)$ and $\left(\mathrm{m}_{1}\right)$. Let there exists a locally Lipschitzian bounding function $V: E \rightarrow \mathbb{R}$ of (3.1) with $K:=r B$ and $r$ as in $\left(\mathrm{m}_{0}\right)$.

Then problem (3.1)-(3.3) admits at least one mild solution $x \in C([a, b], r \bar{B})$.

Proof. The proof splits into two parts. By means of the homotopic invariance of the LeraySchauder degree, we first solve an initial value problem associated to equation (3.1) in an arbitrary interval $\left[a+\frac{1}{m}, b\right]$ with $m \in \mathbb{N}$ sufficiently large (see (3.7)-(3.8) with $\lambda=1$ ). The compactness of the semigroup $S(t)$ is fundamental in this reasoning and this is why we restrict to the interval $\left[a+\frac{1}{m}, b\right]$. In such a way we get a sequence $\left\{x_{m}\right\}$ of continuous functions taking values in $r \bar{B}$. Then we obtain a solution of the original problem (3.1)-(3.3) by passing to the limit in the sequence $\left\{x_{m}\right\}$. Let $Q:=C([a, b], r \bar{B})$ with $r>0$ as in $\left(m_{0}\right)$ and $m \in \mathbb{N}$ such that $a+\frac{1}{m}<b$. Notice that, since $\{S(t)\}_{t \geq 0}$ is a semigroup of contractions, it follows that $S(t) r \bar{B} \subseteq r \bar{B}, t \geq 0$.

STEP 1. We define the map $\mathcal{T}_{m}: Q \times[0,1] \rightarrow C([a, b], E)$ as follows

$$
\mathcal{T}_{m}(q, \lambda)(t)= \begin{cases}\lambda S\left(\frac{1}{m}\right) M(q) & t \in\left[a, a+\frac{1}{m}\right] \\ \lambda S(t-a) M(q)+\lambda \int_{a+\frac{1}{m}}^{t} S(t-s) f(s, q(s)) d s & t \in\left[a+\frac{1}{m}, b\right],\end{cases}
$$

which, according to $(A)$ and $(f)$, is well defined.

With $q, \lambda$ and $m$ as before, let $y_{q, \lambda}, \eta_{q, \lambda}:\left[a+\frac{1}{m}, b\right] \rightarrow E$ be given by

$$
y_{q, \lambda}(t):=\lambda S(t-a) M(q), \quad \eta_{q, \lambda}(t):=\lambda \int_{a+\frac{1}{m}}^{t} S(t-s) f(s, q(s)) d s ;
$$

notice that

$$
\mathcal{T}_{m}(q, \lambda)(t)=y_{q, \lambda}(t)+\eta_{q, \lambda}(t), \quad t \in\left[a+\frac{1}{m}, b\right]
$$

By the equality

$$
S\left(t-\frac{1}{m}-a\right)\left[\lambda S\left(\frac{1}{m}\right) M(q)\right]=\lambda S(t-a) M(q), \quad t \in\left[a+\frac{1}{m}, b\right]
$$

we obtain that the function $x:=\mathcal{T}_{m}(q, \lambda)$ is the unique solution (see [29, Corollary 2.2 p.106]) of the linear initial value problem

$$
\left\{\begin{aligned}
z^{\prime}(t) & =A z(t)+\lambda f(t, q(t)), \quad t \in\left[a+\frac{1}{m}, b\right] \\
z\left(a+\frac{1}{m}\right) & =\lambda S\left(\frac{1}{m}\right) M(q) .
\end{aligned}\right.
$$

In particular, every fixed point $x=\mathcal{T}_{m}(x, \lambda)$, with $x \in Q$ and $\lambda \in[0,1]$ is a mild solution of the equation

$$
x^{\prime}(t)=A x(t)+\lambda f(t, x(t)), \quad t \in\left[a+\frac{1}{m}, b\right]
$$

(see Definition 3.1) which satisfies

$$
x\left(a+\frac{1}{m}\right)=\lambda S\left(\frac{1}{m}\right) M(x) .
$$

We will show that $\mathcal{T}_{m}(\cdot, 1)$ has a fixed point $x_{m}=\mathcal{T}_{m}\left(x_{m}, 1\right)$, with $x_{m} \in Q$. 
The use of a topological method then arises quite naturally and hence we investigate, in the following, the regularity properties of the map $\mathcal{T}_{m}$.

(1a) First we show that $\mathcal{T}_{m}$ is continuous. In fact, let $\left\{q_{n}\right\} \subset Q$ satisfying $q_{n} \rightarrow q$ in $C([a, b], E)$ and let $\left\{\lambda_{n}\right\} \subset[0,1]$ with $\lambda_{n} \rightarrow \lambda$. For every $n \in \mathbb{N}$, the function $x_{n}:=$ $\mathcal{T}_{m}\left(q_{n}, \lambda_{n}\right)$ is such that

$$
x_{n}(t)= \begin{cases}\lambda_{n} S\left(\frac{1}{m}\right) M\left(q_{n}\right), & t \in\left[a, a+\frac{1}{m}\right] \\ y_{q_{n}, \lambda_{n}}(t)+\eta_{q_{n}, \lambda_{n}}(t) & t \in\left[a+\frac{1}{m}, b\right]\end{cases}
$$

(see (3.5)). Put $x:=\mathcal{T}_{m}(q, \lambda)$. Since, by $(A),\|S(t)\| \leq 1$ for $t \geq 0$, when $t \in\left[a, a+\frac{1}{m}\right]$ we have that

$$
\begin{aligned}
\left\|x_{n}(t)-x(t)\right\| & =\left\|\lambda_{n} S\left(\frac{1}{m}\right) M\left(q_{n}\right)-\lambda S\left(\frac{1}{m}\right) M(q)\right\| \\
& \leq \mid \lambda_{n}-\lambda\left\|S\left(\frac{1}{m}\right) M\left(q_{n}\right)\right\|+\lambda\left\|S\left(\frac{1}{m}\right)\left[M\left(q_{n}\right)-M(q)\right]\right\| \\
& \leq\left|\lambda_{n}-\lambda\right|\left\|M\left(q_{n}\right)\right\|+\lambda\left\|M\left(q_{n}\right)-M(q)\right\| .
\end{aligned}
$$

Since $M\left(q_{n}\right) \rightarrow M(q)$ in $E$ (see Remark $\underline{3.1}$ ) hence, in particular, $\left\{M\left(q_{n}\right)\right\}$ is bounded in $E$, we obtain that $x_{n} \rightarrow x$ in $C\left(\left[a, a+\frac{1}{m}\right], E\right)$.

Similarly it is easy to prove that

$$
y_{q_{n}, \lambda_{n}} \rightarrow y_{q, \lambda} \text { in } C\left(\left[a+\frac{1}{m}, b\right], E\right) .
$$

Notice that, by (f) and the convergence of $\left\{q_{n}\right\}$ to $q$, it follows that $f\left(t, q_{n}(t)\right) \rightarrow$ $f(t, q(t)), t \in[a, b]$. Again by $(f)$ the convergence is also dominated since

$$
\left\|f\left(t, q_{n}(t)\right)\right\| \leq \nu_{r \bar{B}}(t), \quad \text { for a.a. } t \in[a, b] .
$$

By the Lebesgue dominated convergence theorem we conclude that, for every $t \in\left[a+\frac{1}{m}, b\right]$,

$$
\begin{aligned}
\left\|\eta_{q_{n}, \lambda_{n}}(t)-\eta_{q, \lambda}(t)\right\| \leq & \left|\lambda_{n}-\lambda\right| \int_{a+\frac{1}{m}}^{t}\left\|S(t-s) f\left(s, q_{n}(s)\right) d s\right\|+ \\
& \quad \lambda \int_{a+\frac{1}{m}}^{t}\left\|S(t-s)\left[f\left(s, q_{n}(s)\right)-f(s, q(s))\right]\right\| d s \\
\leq & \left|\lambda_{n}-\lambda\right| \int_{a+\frac{1}{m}}^{b} \nu_{r \bar{B}}(s) d s+\lambda \int_{a+\frac{1}{m}}^{b}\left\|f\left(s, q_{n}(s)\right)-f(s, q(s))\right\| d s \rightarrow 0 .
\end{aligned}
$$

Hence $\eta_{q_{n}, \lambda_{n}} \rightarrow \eta_{q, \lambda}$ in $C\left(\left[a+\frac{1}{m}, b\right], E\right)$ and by (3.10) this proves that $x_{n} \rightarrow x$ in $C([a, b], E)$, i.e. $\mathcal{T}_{m}$ is a continuous operator.

(1b) Now we show that $\mathcal{T}_{m}$ is compact. To this aim consider a sequence $\left\{x_{n}\right\} \subset \mathcal{T}_{m}(Q \times$ $[0,1])$ which implies the existence of $\left\{q_{n}\right\} \subset Q$ and $\left\{\lambda_{n}\right\} \subset[0,1]$ such that $x_{n}=\mathcal{T}_{m}\left(q_{n}, \lambda_{n}\right)$, i.e. $x_{n}$ satisfies condition (3.9), for all $n \in \mathbb{N}$. With no loss of generality we can restrict to a subsequence, as usual denoted as the sequence, such that $\lambda_{n} \rightarrow \lambda \in[0,1]$.

First consider the interval $\left[a, a+\frac{1}{m}\right]$. Since, by $\left(m_{0}\right)$, the sequence $\left\{M\left(q_{n}\right)\right\} \subset r \bar{B}$ is bounded and the semigroup $\{S(t)\}_{t \geq 0}$ is compact, we obtain that $x_{n}$ is relatively compact in $C([a, a+$ $\left.\left.\frac{1}{m}\right], E\right)$.

Let $t \in\left[a+\frac{1}{m}, b\right]$. As before the set $\left\{y_{q_{n}, \lambda_{n}}(t)\right\} \subset E$ is relatively compact in $E$. We prove now that $\left\{y_{q_{n}, \lambda_{n}}\right\}$ is equicontinuous in $\left[a+\frac{1}{m}, b\right]$. Let, in fact, $t \in\left[a+\frac{1}{m}, b\right]$ and $\varepsilon>0$. The compactness of $\{S(t)\}_{t \geq 0}$ implies the continuity of $S:(0, \infty) \rightarrow \mathcal{L}(E)$ in the uniform operator topology (see [29, Theorem 3.2 p. 49]). Hence we can find $\delta=\delta(\varepsilon)>0$ satisfying

$$
\left\|S(t-a)-S\left(t^{\prime}-a\right)\right\| \leq \frac{\varepsilon}{r}, \quad\left|t-t^{\prime}\right|<\delta \text { with } t^{\prime} \in\left[a+\frac{1}{m}, b\right] .
$$


Therefore, by $\left(m_{0}\right)$

$$
\left\|y_{q_{n}, \lambda_{n}}(t)-y_{q_{n}, \lambda_{n}}\left(t^{\prime}\right)\right\| \leq \lambda_{n}\left\|S(t-a)-S\left(t^{\prime}-a\right)\right\|\left\|M\left(q_{n}\right)\right\| \leq \frac{\varepsilon}{r} \cdot r=\varepsilon,
$$

for every $n \in \mathbb{N}$ and $t, t^{\prime} \in\left[a+\frac{1}{m}, b\right]$ with $\left|t-t^{\prime}\right|<\delta$.

Then, by the abstract version of the Ascoli-Arzelá theorem, we obtain that

$$
\left\{y_{q_{n}, \lambda_{n}}\right\} \text { is relatively compact in } C\left(\left[a+\frac{1}{m}, b\right], E\right) .
$$

Consider now the sequence $\left\{\eta_{q_{n}, \lambda_{n}}\right\}$ defined in (3.5). Fix $t \in\left[a+\frac{1}{m}, b\right]$ and let $h_{n, t}(s):=S(t-s) f\left(s, q_{n}(s)\right)$ for $n \in \mathbb{N}$ and $s \in\left[a+\frac{1}{m}, t\right]$. According to (3.11) and since, by $(A),\|S(t)\| \leq 1$ for $t \geq 0$, we have that $\left\|h_{n, t}(s)\right\| \leq \nu_{r \bar{B}}(s)$ for a.a. $s \in\left[a+\frac{1}{m}, t\right]$, hence $\left\{h_{n, t}\right\}$ is integrably bounded in $\left[a+\frac{1}{m}, t\right]$. Moreover, condition (3.11) implies that $\left\{f\left(s, q_{n}(s)\right)\right\}$ is bounded in $E$ for a.a. $s \in\left[a+\frac{1}{m}, t\right]$ and by the compactness of the semigroup $\{S(t)\}_{t \geq 0}$ we obtain that the sequence $\left\{h_{n, t}(s)\right\}$ is relatively compact for a.a. $s \in\left[a+\frac{1}{m}, t\right]$. In conclusion the sequence $\left\{h_{n, t}\right\}$ is semicompact in $\left[a+\frac{1}{m}, t\right]$ (see Definition 2.2).

Let us introduce now the operator $\hat{S}: L^{1}\left(\left[a+\frac{1}{m}, t\right], E\right) \rightarrow C\left(\left[a+\frac{1}{m}, t\right], E\right)$ given by

$$
\hat{S} \varphi(\tau):=\int_{a+\frac{1}{m}}^{\tau} \varphi(s) d s
$$

It is easy to see that $\hat{S}$ satisfies both conditions (i) and (ii) of Theorem 2.2. indeed $\hat{S}$ is the Cauchy operator (see Example 2.1) in the special case when the semigroup is identically equal to $I$. By virtue of Theorem 2.2 , the sequence $\left\{\eta_{q_{n}, \lambda_{n}}\right\}$ is relatively compact in $C\left(\left[a+\frac{1}{m}, t\right], E\right)$. Hence, in particular, $\left\{\eta_{q_{n}, \lambda_{n}}(t)\right\}$ is relatively compact in $E$ for all $t \in\left[a+\frac{1}{m}, b\right]$.

We prove now that $\left\{\eta_{q_{n}, \lambda_{n}}\right\}$ is equicontinuous. In fact, fix $t \in\left[a+\frac{1}{m}, b\right]$ and let $t^{\prime} \in\left[a+\frac{1}{m}, b\right]$ with $t^{\prime}>t$. Notice that

$$
\left\|\eta_{q_{n}, \lambda_{n}}\left(t^{\prime}\right)-\eta_{q_{n}, \lambda_{n}}(t)\right\|=\lambda_{n}\left\|\int_{a+\frac{1}{m}}^{t^{\prime}} S\left(t^{\prime}-s\right) f\left(s, q_{n}(s)\right) d s-\int_{a+\frac{1}{m}}^{t} S(t-s) f\left(s, q_{n}(s)\right) d s\right\| .
$$

For every given $\sigma \in\left(0, t-\frac{1}{m}-a\right)$, we can then estimate $\left\|\eta_{q_{n}, \lambda_{n}}\left(t^{\prime}\right)-\eta_{q_{n}, \lambda_{n}}(t)\right\|$ by means of the sum of the following three integrals

$$
\begin{aligned}
\left\|\eta_{q_{n}, \lambda_{n}}\left(t^{\prime}\right)-\eta_{q_{n}, \lambda_{n}}(t)\right\| & \leq \lambda_{n}\left\|\int_{a+\frac{1}{m}}^{t-\sigma}\left[S\left(t^{\prime}-s\right)-S(t-s)\right] f\left(s, q_{n}(s)\right) d s\right\| \\
& +\lambda_{n}\left\|\int_{t-\sigma}^{t}\left[S\left(t^{\prime}-s\right)-S(t-s)\right] f\left(s, q_{n}(s)\right) d s\right\| \\
& +\lambda_{n}\left\|\int_{t}^{t^{\prime}} S\left(t^{\prime}-s\right) f\left(s, q_{n}(s)\right) d s\right\| .
\end{aligned}
$$

Now fix $\varepsilon>0$. With no loss of generality we can take $\sigma>0$ such that

$$
\int_{t-\sigma}^{t} \nu_{r \bar{B}}(s) d s<\frac{\varepsilon}{6}
$$


Let us start from the first integral in (3.13). Notice that $t^{\prime}-s, t-s \in\left[\sigma, t^{\prime}-\frac{1}{m}-a\right]$ for $s \in\left[a+\frac{1}{m}, t-\sigma\right]$. Since $\{S(t)\}_{t \geq 0}$ is compact, it is also uniformly continuous in the compact interval $\left[\sigma, t^{\prime}-\frac{1}{m}-a\right]$. We can then find $\sigma_{1}(\varepsilon)>0$ such that

$$
\left\|S\left(\tau^{\prime}\right)-S(\tau)\right\| \leq \frac{\varepsilon}{3\left\|\nu_{r \bar{B}}\right\|}, \quad\left|\tau^{\prime}-\tau\right|<\sigma_{1}, \quad \tau^{\prime}, \tau \in\left[\sigma, t^{\prime}-\frac{1}{m}-a\right] .
$$

Since $t^{\prime}-s-(t-s)=t^{\prime}-t$ for $s \in\left[a+\frac{1}{m}, t-\sigma\right]$, when $0<t^{\prime}-t<\sigma_{1}$, by (3.11) and since $\|S(t)\| \leq 1$ for $t \geq 0$, we have

$$
\begin{aligned}
\lambda_{n}\left\|\int_{a+\frac{1}{m}}^{t-\sigma}\left[S\left(t^{\prime}-s\right)-S(t-s)\right] f\left(s, q_{n}(s)\right) d s\right\| & \leq \int_{a+\frac{1}{m}}^{t-\sigma}\left\|S\left(t^{\prime}-s\right)-S(t-s)\right\|\left\|f\left(s, q_{n}(s)\right)\right\| d s \\
& \leq \frac{\varepsilon}{3\left\|\nu_{r \bar{B}}\right\|} \int_{a}^{b}\left\|f\left(s, q_{n}(s)\right)\right\| d s \leq \frac{\varepsilon}{3} .
\end{aligned}
$$

According to (3.14) the second integral in (3.13) is such that

$$
\begin{aligned}
\lambda_{n}\left\|\int_{t-\sigma}^{t}\left[S\left(t^{\prime}-s\right)-S(t-s)\right] f\left(s, q_{n}(s)\right) d s\right\| & \leq \int_{t-\sigma}^{t}\left[\left\|S\left(t^{\prime}-s\right)\right\|+\|S(t-s)\|\right]\left\|f\left(s, q_{n}(s)\right)\right\| d s \\
& \leq 2 \int_{t-\sigma}^{t} \nu_{r \bar{B}}(s) d s \leq \frac{\varepsilon}{3} .
\end{aligned}
$$

Moreover, let $\sigma_{2}>0$ be such that

$$
\int_{t}^{t^{\prime}} \nu_{r \bar{B}}(s) d s \leq \frac{\varepsilon}{3}, \quad \text { for } t^{\prime}-t<\sigma_{2}
$$

hence we obtain the following estimate for the third integral in (3.13)

$$
\lambda_{n}\left\|\int_{t}^{t^{\prime}} S\left(t^{\prime}-s\right) f\left(s, q_{n}(s)\right) d s\right\| \leq \int_{t}^{t^{\prime}} \nu_{r \bar{B}}(s) d s \leq \frac{\varepsilon}{3} .
$$

Consequently, when $t^{\prime}-t<\min \left\{\sigma_{1}, \sigma_{2}\right\}$, we have that $\left\|\eta_{q_{n}, \lambda_{n}}\left(t^{\prime}\right)-\eta_{q_{n}, \lambda_{n}}(t)\right\|<\varepsilon$; since the reasoning is similar also in the case $t^{\prime} \in\left[a+\frac{1}{m}, t\right]$ we conclude that $\left\{\eta_{q_{n}, \lambda_{n}}\right\}$ is equicontinuous in $\left[a+\frac{1}{m}, b\right]$ and again we can use the abstract version of Arzelà-Ascoli theorem in order to show that $\left\{\eta_{q_{n}, \lambda_{n}}\right\}$ is relatively compact in $C\left(\left[a+\frac{1}{m}, b\right], E\right)$. Therefore, by (3.12) and the estimates in the interval $\left[a, a+\frac{1}{m}\right]$ (see the beginning of $(1 \mathrm{~b})$ ), we have that $\left\{x_{n}\right\}$ is relatively compact and so the operator $\mathcal{T}_{m}$ is compact. In conclusion $\mathcal{T}_{m}$ is completely continuous since it is both continuous and compact.

(1c) For every $q \in Q$ we have that $\mathcal{T}_{m}(q, 0) \equiv 0$ and since $0 \in r B$, it implies that $\mathcal{T}_{m}(Q, 0) \subset \operatorname{int} Q$.

(1d) We apply now a degree argument for the study of the fixed points of $\mathcal{T}_{m}(\cdot, 1)$ and then we need to show that $\mathcal{T}_{m}(\cdot, \lambda)$ is fixed points free on $\partial Q$ for every $\lambda \in[0,1]$. The case $\lambda=0$ was already treated in (1c). Any possible fixed point $x \in \partial Q$ for $\lambda=1$, i.e. satisfying $x=\mathcal{T}_{m}(x, 1)$, is already a solution of our problem. So, it remains to show that $\mathcal{T}_{m}(\cdot, \lambda)$ is fixed-points free on $\partial Q$ only for $\lambda \in(0,1)$. We reason by contradiction and assume the existence of $(x, \lambda) \in \partial Q \times(0,1)$ satisfying $x=\mathcal{T}_{m}(x, \lambda)$. According to the definition of $Q$, there exists $t_{0} \in[a, b]$ such that $\left\|x\left(t_{0}\right)\right\|=r$. By $\left(m_{0}\right)$ and since $\{S(t)\}_{t \geq 0}$ is a semigroup of contractions, the case $t_{0} \in\left[a, a+\frac{1}{m}\right]$ leads to the contradictory conclusion

$$
r=\left\|x\left(t_{0}\right)\right\|=\lambda\left\|S\left(\frac{1}{m}\right) M(x)\right\| \leq \lambda r<r .
$$


Consequently $t_{0} \in\left(a+\frac{1}{m}, b\right]$; we recall that $x(t)$ is a mild solution of the equation

$$
x^{\prime}(t)=A x(t)+h(t, x(t)), \quad t \in\left[a+\frac{1}{m}, b\right]
$$

where

$$
h(t, x):=\lambda f(t, x), \quad(t, x) \in[a, b] \times E .
$$

Notice that $h$ satisfies conditions $(f)$. We show that $V$ is a bounding function for (3.15) with $K=r B$, in particular that $V$ satisfies condition (V2). In fact, let $x \in E$ with $\|x\|=r$ and $t \in(a, b]$; since $V$ is a bounding function for (3.1) there is a sequence $\left\{h_{n}\right\} \subset \mathbb{R}$, with $h_{n} \rightarrow 0^{-}$such that

$$
\lim _{n \rightarrow \infty} \frac{V\left(x+h_{n} f(t, x)\right)}{h_{n}}<0 .
$$

Put $k_{n}:=\frac{h_{n}}{\lambda}, n \in \mathbb{N}$; we have that $k_{n} \rightarrow 0^{-}$and

$$
\lim _{n \rightarrow \infty} \frac{V\left(x+k_{n} g(t, x)\right)}{k_{n}}=\lim _{n \rightarrow \infty} \frac{V\left(x+k_{n} \lambda f(t, x)\right)}{k_{n}}=\lambda \lim _{n \rightarrow \infty} \frac{V\left(x+h_{n} f(t, x)\right)}{h_{n}}<0 .
$$

Hence $V$ is a bounding function for (3.15) and by applying Theorem 5.1 to (3.15) we obtain that $\left\|x\left(t_{0}\right)\right\|<r$. In conclusion $\|x(t)\|<r$ for all $t \in\left[a+\frac{1}{m}, b\right]$ and hence $\mathcal{T}_{m}$ is fixed points free on the boundary $\partial Q$.

(1e) The properties already proved imply that $\mathcal{T}_{m}$ is an admissible homotopy which connects the fields $\mathcal{T}_{m}(\cdot, 0)$ and $\mathcal{T}_{m}(\cdot, 1)$. According to the homotopic invariance and the normalization property of the Leray-Schauder topological degree, we then obtain

$$
\operatorname{deg}\left(i-\mathcal{T}_{m}(\cdot, 1), Q\right)=\operatorname{deg}\left(i-\mathcal{T}_{m}(\cdot, 0), Q\right)=1
$$

and hence there exists $x_{m} \in Q$ such that $x_{m}=\mathcal{T}_{m}\left(x_{m}, 1\right)$, i.e. $x_{m}(t) \equiv S\left(\frac{1}{m}\right) M\left(x_{m}\right)$ for $t \in\left[a, a+\frac{1}{m}\right]$ and $x_{m}$ is a solution of the initial value problem (3.7)-(3.8) for $\lambda=1$ on $\left[a+\frac{1}{m}, b\right]$; moreover $\left\|x_{m}(t)\right\| \leq r$ for $t \in[a, b]$ with $r$ introduced in $\left(m_{0}\right)$.

STEP 2. In this part we consider the sequence of functions $\left\{x_{m}\right\}$ obtained in the previous step and, by passing to the limit, we get a solution of problem (3.1)-(3.3). According to (3.4), we recall that

$$
x_{m}(t)=\left\{\begin{aligned}
S\left(\frac{1}{m}\right) M\left(x_{m}\right) & t \in\left[a, a+\frac{1}{m}\right] \\
S(t-a) M\left(x_{m}\right)+\int_{a+\frac{1}{m}}^{t} S(t-s) f\left(s, x_{m}(s)\right) d s, & t \in\left[a+\frac{1}{m}, b\right] .
\end{aligned}\right.
$$

(2a) Take $\alpha \in(a, b]$ and let $m$ be sufficiently large so that $a+\frac{1}{m}<\alpha$. Since $\left\{x_{m}\right\} \subset Q$, according to $(f)$ we have that $\left\|f\left(t, x_{m}(t)\right)\right\| \leq \nu_{r \bar{B}}(t)$ for a.a. $t \in[a, b]$. Hence, with a similar reasoning as in $(1 b)$, we can show that $\left\{x_{m}\right\}$ is relatively compact in $[\alpha, b]$.

(2b) Fix a decreasing sequence $\left\{a_{n}\right\} \subset(a, b)$ satisfying $a_{n} \rightarrow a$ as $n \rightarrow \infty$. According to (2a), $\left\{x_{m}\right\}$ is relatively compact in $C\left(\left[a_{1}, b\right], E\right)$; hence there is a subsequence $\left\{x_{m}^{(1)}\right\}$ converging in $C\left(\left[a_{1}, b\right], E\right)$ to a continuous function $\bar{x}:\left[a_{1}, b\right] \rightarrow E$. Similarly, there exists a subsequence $\left\{x_{m}^{(2)}\right\}$ of $\left\{x_{m}^{(1)}\right\}$ converging in $C\left(\left[a_{2}, b\right], E\right)$ to a continuous function 
$\overline{\bar{x}}:\left[a_{2}, b\right] \rightarrow E$ and, according to the unicity of the limit, $\bar{x}(t)=\overline{\bar{x}}(t)$ for $t \in\left[a_{1}, b\right]$. Proceeding by induction, for every $n \in \mathbb{N}$ we can find a sequence $\left\{x_{m}^{(n)}\right\}$ which is a subsequence of $\left\{x_{m}^{(n-1)}\right\}$ and converges in $C\left(\left[a_{n}, b\right], E\right)$. According to the unicity of the limit, we can define a continuous function $\tilde{x}:(a, b] \rightarrow E$ and, using a Cantor diagonalization argument, the sequence $x_{n}^{(n)}(t) \rightarrow \tilde{x}(t)$ for $t \in(a, b]$. By the continuity of $f$ (see condition $(f)$ ), it implies that $f\left(t, x_{n}^{(n)}(t)\right) \rightarrow f(t, \tilde{x}(t))$ for all $t \in(a, b]$ and also that $t \longmapsto f(t, \tilde{x}(t))$ is continuous on $(a, b]$. Moreover, since $\left\{x_{m}\right\} \subset Q$, again by $(f)$ we have that $\left\|f\left(t, x_{n}^{(n)}(t)\right)\right\| \leq \nu_{r} \bar{B}(t)$ and hence $\|f(t, \tilde{x}(t))\| \leq \nu_{r \bar{B}}(t)$ for a.a. $t \in(a, b]$, with $\nu_{r \bar{B}} \in L^{1}([a, b])$.

(2c) Since $\left\{x_{n}^{(n)}\right\} \subset C([a, b], r \bar{B})$, by $\left(m_{0}\right)$ we obtain that $\left\{M\left(x_{n}^{(n)}\right)\right\} \subset r \bar{B}$. Hence, by the reflexivity of $E$, there is a subsequence, as usual denoted as the sequence, satisfying

$$
M\left(x_{n}^{(n)}\right) \rightarrow x_{0} \text { as } n \rightarrow \infty, \text { with }\left\|x_{0}\right\| \leq r .
$$

(2d) Let us introduce, now, the continuous function

$$
x(t):=S(t-a) x_{0}+\int_{a}^{t} S(t-s) f(s, \tilde{x}(s)) d s, \quad t \in[a, b],
$$

with $x_{0}$ as in (2c) and $\tilde{x}$ defined in (2b). The integral in (3.19) is well defined by the regularity of the function $t \longmapsto f(t, \tilde{x}(t))$ in $[a, b]$ showed in (2b). We claim that

$$
x_{n}^{(n)}(t) \rightarrow x(t), \quad t \in(a, b], \quad \text { as } n \rightarrow \infty .
$$

In fact, by (3.17) and the definition of $\left\{x_{n}^{(n)}\right\}$, there is a sequence $\left\{p_{n}\right\}$, with $0<p_{n} \leq$ $1 / n, n \in \mathbb{N}$, such that

$$
x_{n}^{(n)}(t)= \begin{cases}S\left(p_{n}\right) M\left(x_{n}^{(n)}\right) & t \in\left[a, a+p_{n}\right] \\ S(t-a) M\left(x_{n}^{(n)}\right)+\int_{a+p_{n}}^{t} S(t-s) f\left(s, x_{n}^{(n)}(s)\right) d s & t \in\left[a+p_{n}, b\right] .\end{cases}
$$

Let $t \in(a, b]$. By (3.18) we have that

$$
S(t-a) M\left(x_{n}^{(n)}\right) \rightarrow S(t-a) x_{0} .
$$

Now take $n \geq \bar{n}$ such that $t \in\left[a+p_{n}, b\right]$ for every $n \geq \bar{n}$. Notice that

$$
\int_{a+p_{n}}^{t} S(t-s) f\left(s, x_{n}^{(n)}(s)\right) d s=\int_{a}^{t} S(t-s) f\left(s, x_{n}^{(n)}(s)\right) d s-\int_{a}^{a+p_{n}} S(t-s) f\left(s, x_{n}^{(n)}(s)\right) d s .
$$

By the properties showed in (2b), we have

$$
S(t-s) f\left(s, x_{n}^{(n)}(s)\right) \rightarrow S(t-s) f(s, \tilde{x}(s)), s \in(a, t] .
$$

Moreover $\left\|S(t-s) f\left(s, x_{n}^{(n)}(s)\right)\right\| \leq \nu_{r \bar{B}}(s)$ a.e. in $[a, t]$, with $\nu_{r \bar{B}} \in L^{1}([a, t])$. So the Lebesgue Dominated Convergence Theorem leads to

$$
\int_{a}^{t} S(t-s) f\left(s, x_{n}^{(n)}(s)\right) d s \rightarrow \int_{a}^{t} S(t-s) f(s, \tilde{x}(s)) d s, \text { as } n \rightarrow \infty .
$$


Since $0<p_{n} \leq 1 / n, n \in \mathbb{N}$, we have

$$
\int_{a}^{a+p_{n}} S(t-s) f\left(s, x_{n}^{(n)}(s)\right) d s \rightarrow 0 \text { as } n \rightarrow \infty ;
$$

hence, by (3.21),$x_{n}^{(n)}(t) \rightarrow x(t)$. Since we showed in (2b) that $x_{n}^{(n)}(t) \rightarrow \tilde{x}(t)$ for $t \in(a, b]$, we obtain that $\tilde{x}(t)=x(t)$ and hence, by (3.19), $x$ is a mild solution of (3.1) in $[a, b]$. By condition $\left(m_{1}\right)$ we get that $M\left(x_{n}^{(n)}\right) \rightarrow M(x)$ thus (3.18) implies that $x(a)=x_{0}=M(x)$ and hence $x$ satisfies the boundary condition (3.3). The proof is complete.

\section{Nonlocal solutions of the parabolic equation (1.1)}

In this part we apply the methods and results contained in Section 3 for the study of nonlocal boundary value problems associated to equation (1.1). We prove, in particular, Theorem 1.1 as a special case of the following more general result (see Theorem 4.1).

We put

$$
\mu:=\max _{t \in[0, T]}|g(t, 0)| .
$$

The definition is well posed by the continuity of $g$ (see condition (1.5) (i)).

Theorem 4.1. Consider problem (1.1)-(1.4)-(1.11). Let $a_{i, j} \in C^{1}(\bar{D}), i, j=1, \ldots, n$ satisfy conditions (1.2), (1.3) and assume (1.5); suppose $\left(m_{0}\right)$ and $\left(m_{1}\right)$ hold (see Section 3 ) with $E=L^{p}(D)$. If, moreover,

(a) $b>L+|D|$

(b) $r>\frac{(L+\mu)|D|}{b-|D|-L}$,

with $\mu$ as in (4.1), then (1.1)-(1.4)-(1.11) is solvable.

Proof. We reformulate (1.1)-(1.4)-(1.11) in the abstract setting (3.1)-(3.3) where $E:=$ $L^{p}(D), 1<p<\infty$.

The linear elliptic partial differential operator in divergence form $A_{p}$ introduced in (1.6) generates a compact $C_{0}$-semigroup of contractions $\{S(t)\}_{t \geq 0}$ (see Theorem 2.1).

Given $\eta \in E$ we denote, as usual, with $|\eta|$ the map $\xi \longmapsto|\eta(\xi)|$ for a.a. $\xi \in D$. Consider $\beta \in(0,1)$; since $D$ is bounded and $c>0$ implies $c^{\beta} \leq \max \{1, c\}$, it is clear that also $|\eta|^{\beta} \in E$. Moreover it is easy to see that $|\eta|^{p \beta} \in L^{1 / \beta}(D)$ and the following estimate is satisfied

$$
\left\||\eta|^{\beta}\right\| \leq|D|^{\frac{1-\beta}{p}} \cdot\|\eta\|^{\beta}, \quad \eta \in E .
$$

We introduce the function $f:[0, T] \times E \rightarrow E$ defined by

$$
f(t, \eta)(\xi):=\int_{D} k(\xi, y) \eta(y) d y-b \eta(\xi)+g(t, \eta(\xi)), \text { for a.a. } \xi \in D .
$$

By (1.5) we have

$$
|f(t, \eta)(\xi)| \leq \int_{D}|\eta(y)| d y+b|\eta(\xi)|+|g(t, \eta(\xi))-g(t, 0)|+|g(t, 0)|,
$$


for $t \in[0, T]$ and a.a. $\xi \in D$. Hence, by the Hölder inequality, we obtain

$$
\begin{aligned}
|f(t, \eta)(\xi)| \leq & |D|^{1-\frac{1}{p}}\|\eta\|+b|\eta(\xi)|+L \max \left\{|\eta(\xi)|,|\eta(\xi)|^{\beta}\right\}+\mu \\
& |D|^{1-\frac{1}{p}}\|\eta\|+(b+L)|\eta(\xi)|+L+\mu, \text { for a.a. } \xi \in D
\end{aligned}
$$

with $\mu$ as in (4.1), implying that $f$ is well-defined. Therefore, in abstract setting, equation (1.1) takes the form (3.1) with $x(t)=u(t, \cdot)$.

Now we show that $f$ is continuous. Let $\left(t_{n}, \eta_{n}\right) \rightarrow(t, \eta)$ in $[0, T] \times E$. By the continuity of $g$ we have that $g\left(t_{n}, \eta(\xi)\right) \rightarrow g(t, \eta(\xi))$, for a.a. $\xi \in D$ and the convergence is dominated in E since

$$
\begin{aligned}
\left|g\left(t_{n}, \eta(\xi)\right)-g(t, \eta(\xi))\right| \leq & \left|g\left(t_{n}, \eta(\xi)\right)-g\left(t_{n}, 0\right)\right|+|g(t, \eta(\xi))-g(t, 0)| \\
& +\left|g\left(t_{n}, 0\right)\right|+|g(t, 0)| \\
\leq & 2 L(1+|\eta(\xi)|)+2 \mu, \text { for a.a. } \xi \in D .
\end{aligned}
$$

Therefore

$$
\left\|g\left(t_{n}, \eta(\cdot)\right)-g(t, \eta(\cdot))\right\| \rightarrow 0, \text { as } n \rightarrow \infty, \text { in } E .
$$

By (1.5) and the Hölder inequality, we have

$$
\begin{aligned}
\left|f\left(t_{n}, \eta_{n}\right)(\xi)-f(t, \eta)(\xi)\right| \leq & \left|f\left(t_{n}, \eta_{n}\right)(\xi)-f\left(t_{n}, \eta\right)(\xi)\right|+\left|f\left(t_{n}, \eta\right)-f(t, \eta)\right|(\xi) \\
\leq & |D|^{1-\frac{1}{p}}\left\|\eta_{n}-\eta\right\|+(b+L)\left|\eta_{n}(\xi)-\eta(\xi)\right|+L\left|\eta_{n}(\xi)-\eta(\xi)\right|^{\beta} \\
& +\left|g\left(t_{n}, \eta(\xi)\right)-g(t, \eta(\xi))\right| .
\end{aligned}
$$

Hence, by (4.2) and (4.5), $f\left(t_{n}, \eta_{n}\right) \rightarrow f(t, \eta)$ in $E$ and $f$ is continuous.

We prove that $f$ satisfies also the growth condition in $(f)$. So, let $\Omega \subset E$ be bounded and take $\eta \in \Omega$. By the estimate

$$
(a+b)^{p} \leq 2^{p}\left(a^{p}+b^{p}\right) \quad \text { for } a, b \geq 0 \text { and } p>1,
$$

and according to (4.4) we have

$$
\|f(t, \eta)\|^{p}=\int_{D}[|f(t, \eta)|(\xi)]^{p} d \xi \leq 2^{p}(b+L)^{p}\|\eta\|^{p}+2^{p}\left(|D|^{1-\frac{1}{p}}\|\eta\|+L+\mu\right)^{p}|D|,
$$

for all $t \in[0, T]$ and hence $(f)$ is satisfied.

By assumption, the nonlocal condition (1.11) satisfies both $\left(m_{0}\right)$ and $\left(m_{1}\right)$.

It remains to show the existence of a locally Lipschitzian bounding function $V: E \rightarrow \mathbb{R}$ (Definition 3.2) with $K=r B$ and $r$ as in $\left(m_{0}\right)$. Consider the function

$$
V_{r}(x)=\frac{1}{2}\left(\|x\|^{2}-r^{2}\right), \quad x \in E .
$$

$V_{r}$ is locally Lipschitzian; it is also Fréchet differentiable, since $L^{p}(D)$ is uniformly convex, and

$$
\left\langle\dot{V}_{r}(y), z\right\rangle=\frac{1}{\|y\|^{p-2}} \int_{D}|y(\xi)|^{p-2} y(\xi) z(\xi) d \xi, \quad y, z \in E
$$

(see e.g. Example 5.1(ii)). We prove that $V_{r}$ satisfies condition (V2). Notice that, since $D$ is bounded,

$$
\int_{D}|\eta(\xi)|^{p-1} d \xi \leq|D|^{\frac{1}{p}}\|\eta\|^{p-1}, \quad \eta \in L^{p}(D)
$$


Therefore, we have the following estimate

$$
\begin{aligned}
& \left.\left|\int_{D}\right| \eta(\xi)\right|^{p-2} \eta(\xi)\left[\int_{D} k(\xi, y) \eta(y) d y+g(t, \eta(\xi))\right] d \xi \mid \\
\leq & \int_{D}|\eta(\xi)|^{p-1}\left(\int_{D}|\eta(y)| d y+|g(t, \eta(\xi))-g(t, 0)|+\mu\right) d \xi \\
\leq & \int_{D}|\eta(\xi)|^{p-1}\left(L|\eta(\xi)|+|D|^{1-\frac{1}{p}}\|\eta\|+L+\mu\right) d \xi \\
\leq & L \int_{D}|\eta(\xi)|^{p} d \xi+\left(|D|^{1-\frac{1}{p}}\|\eta\|+L+\mu\right) \int_{D}|\eta(\xi)|^{p-1} d \xi \\
\leq & L\|\eta\|^{p}+\left(|D|^{1-\frac{1}{p}}\|\eta\|+L+\mu\right)|D|^{\frac{1}{p}}\|\eta\|^{p-1} \\
= & (|D|+L)\|\eta\|^{p}+(L+\mu)|D|^{\frac{1}{p}}\|\eta\|^{p-1} .
\end{aligned}
$$

Thus, if $\|\eta\|=r$, by means of conditions (a)-(b) and the Hölder inequality, we have that

$$
\begin{aligned}
\left\langle\dot{V}_{r}(\eta), f(t, \eta)\right\rangle & =\frac{1}{\|\eta\|^{p-2}} \int_{D}|\eta(\xi)|^{p-2} \eta(\xi)\left[\int_{D} k(\xi, y) \eta(y) d y-b \eta(\xi)+g(t, \eta(\xi))\right] d \xi \\
& =-b\|\eta\|^{2}+\frac{1}{\|\eta\|^{p-2}} \int_{D}|\eta(\xi)|^{p-2} \eta(\xi)\left[\int_{D} k(\xi, y) \eta(y) d y+g(t, \eta(\xi))\right] d \xi \\
& \leq-b\|\eta\|^{2}+\left.\frac{1}{\|\eta\|^{p-2}}\left|\int_{D}\right| \eta(\xi)\right|^{p-2} \eta(\xi)\left[\int_{D} k(\xi, y) \eta(y) d y+g(t, \eta(\xi))\right] d \xi \mid \\
& \leq(-b+|D|+L)\|\eta\|^{2}+(L+\mu)|D|^{\frac{1}{p}}\|\eta\| \\
& =(-b+|D|+L) r^{2}+(L+\mu)|D|^{\frac{1}{p}} r .
\end{aligned}
$$

Since $b>L+|D|$, when

$$
r>\frac{(L+\mu)|D|^{1 / p}}{b-|D|-L}
$$

we obtain that

$$
\left\langle\dot{V}_{r}(\eta), f(t, \eta)\right\rangle<0,\|\eta\|=r
$$

and then $V_{r}$ is a locally Lipschitzian bounding function for (3.1) (see Remark 5.1).

All the assumptions of Theorem 3.1 are then satisfied and the proof is complete

Proof of Theorem 1.1 The proof follows from Theorem 4.1, It remains only to show that (1.9) and (1.10) in the abstract setting satisfy $\left(m_{0}\right)$ and $\left(m_{1}\right)$ in $E=L^{p}(D)$.

(i) Let $M: C\left([0, T], L^{p}(D)\right) \rightarrow L^{p}(D)$ be defined by

$$
M x=\frac{1}{T} \int_{0}^{T} x(t) d t .
$$


Notice that the definition is well-posed since $x(t)$ is a continuous function. Let $r>0$ and consider $x \in C\left([0, T], L^{p}(D)\right)$ with $\|x\| \leq r$. Then

$$
\|M x\| \leq \frac{1}{T} \int_{0}^{T}\|x(t)\| d t \leq r
$$

hence condition $\left(m_{0}\right)$ is satisfied for any $r>0$. With no loss of generality, we can then assume that also condition (b) in Theorem 4.1 is satisfied. Let $\left\{x_{n}\right\} \subset C\left([0, T], L^{p}(D)\right)$ be such that $x_{n}(t) \rightarrow x(t), t \in(0, T]$ with $x \in C\left([0, T], L^{p}(D)\right)$ and $\left\|x_{n}\right\| \leq r$ for all $n$. The convergence of $\left\{x_{n}\right\}$ is then dominated, implying that

$$
M x_{n}=\frac{1}{T} \int_{0}^{T} x_{n}(t) d t \rightarrow \frac{1}{T} \int_{0}^{T} x(t) d t=M x
$$

hence also $\left(m_{1}\right)$ is satisfied. By applying Theorem 4.1, we state claim (i).

(ii) Let $M: C\left([0, T], L^{p}(D)\right) \rightarrow L^{p}(D)$ be such that

$$
M x=\sum_{i=1}^{q} \alpha_{i} x\left(t_{i}\right), \text { with } t_{i} \in(0, T], \alpha_{i} \in \mathbb{R}, i=1, \ldots, q \text { and } \sum_{i=1}^{q}\left|\alpha_{i}\right| \leq 1 .
$$

If $x \in C\left([0, T], L^{p}(D)\right)$ with $\|x\| \leq r, r>0$ we have

$$
\|M x\| \leq \sum_{i=1}^{q}\left|\alpha_{i}\right|\left\|x\left(t_{i}\right)\right\| \leq r \sum_{i=1}^{q}\left|\alpha_{i}\right| \leq r
$$

implying condition $\left(m_{0}\right)$. As in (i), by the arbitrariness of $r$ we can assume that condition (b) in Theorem 4.1 is satisfied. If, moreover, $\left\{x_{n}\right\} \subset C\left([0, T], L^{p}(D)\right)$ and $x \in C\left([0, T], L^{p}(D)\right)$ are defined as in (i), it is easy to see that

$$
M x_{n}=\sum_{i=1}^{q} \alpha_{i} x_{n}\left(t_{i}\right) \rightarrow \sum_{i=1}^{q} \alpha_{i} x\left(t_{i}\right)=M x, \text { as } n \rightarrow \infty,
$$

so also $\left(m_{1}\right)$ is satisfied. Claim (ii) then follows, again by Theorem 4.1, and the proof is complete.

\section{$5 \quad$ Bounding functions for mild solutions}

This part contains a brief discussion about the notion of bounding function introduced in Section 3 (see Definition 3.2). Notice that the set $\bar{K}$ in Definition 3.2 is the 0-sublevel set of $V$. The function $V$ takes its name from its relevant property. In fact, when such a $V$ exists, every solution $x \in C([a, b], E)$ of (3.1) which is located in $\bar{K}$ lies, indeed, in $K$ for $t \in(a, b]$ (see Theorem [5.1). Hence, the existence of a bounding function for (3.1) makes the transversality condition automatically satisfied on $(a, b]$ and it remains to check the behaviour of the solution only for $t=a$.

The bounding function theory was originally introduced in [13] and [26] (see also [14]), in the framework of finite dimensional systems and with smooth bounding function. Again in 
Euclidean spaces, the theory was extended in [30] and in [36], to the case of non-smooth functions. The bounding function theory was developed in 11, in an infinite dimensional setting, when $A: E \rightarrow E$ is linear and bounded and then (3.1) has classical, i.e. absolutely continuous, solutions. A special type of bounding function (see (5.9) below) was used in [3], in combination with the Yoshida approximation of the linear part.

To the best of our knowledge, no result about the existence of bounding functions is available in the present general framework, i.e. in an arbitrary Banach space when the linear term is not necessarily bounded and it generates a $C_{0}$-semigroup.

Remark 5.1. Let us denote with $\langle\cdot, \cdot\rangle$ the duality between $E$ and its dual space $E^{*}$. When $V$ is Gâteaux differentiable it is easy to see that

$$
\lim _{h \rightarrow 0} \frac{V(x+h y)-V(x)}{h}=\langle\dot{V}(x), y\rangle, \quad x, y \in E .
$$

Therefore, since $V(x)=0, x \in \partial K$, if $V$ is Gâteaux differentiable on $\partial K$, condition (V2) simply reduces to the inequality

$$
\langle\dot{V}(x), f(t, x)\rangle<0, \text { for } t \in(a, b] \text { and } x \in \partial K .
$$

Theorem 5.1. Let $E$ be a Banach space, $A: D(A) \subset E \rightarrow E$ linear, not necessarily bounded and such that it generates a $C_{0}$-semigroup $S:[0, \infty) \rightarrow \mathcal{L}(E)$ and $f:[a, b] \times E \rightarrow E$ continuous. Assume the existence of a bounding function $V: E \rightarrow \mathbb{R}$ of (3.1) (Definition 3.2) which is locally Lipschitzian with $K \subset E$ and let there is $\delta \in(0, \infty)$ such that

$$
S(\tau) \bar{K} \subseteq \bar{K} \quad \text { for } \tau \in[0, \delta] .
$$

If $x:[a, b] \rightarrow E$ is a mild solution of (3.1) with $x(t) \in \bar{K}$ for $t \in[a, b]$, then $x(t) \in K$ for $t \in(a, b]$.

Proof. Let $x:[a, b] \rightarrow E$ be a solution of (3.1) satisfying $x(t) \in \bar{K}$ for all $t \in[a, b]$. Assume, by contradiction, the existence of $\hat{t} \in(a, b]$ such that $\hat{x}:=x(\hat{t}) \in \partial K$.

By condition (V2) there is a sequence $\left\{k_{n}\right\} \subset(-\infty, 0)$ with $\hat{t}+k_{n}>a$ for all $n$ such that $k_{n} \rightarrow 0^{-}$as $n \rightarrow \infty$ and

$$
\lim _{n \rightarrow \infty} \frac{V\left(\hat{x}+k_{n} f(\hat{t}, \hat{x})\right)}{k_{n}}<0 .
$$

According to the definition of mild solution (see Definition 3.1) and the properties of the semigroup we have that

$$
\begin{aligned}
\hat{x}=x(\hat{t})= & S(\hat{t}-a) x(a)+\int_{a}^{\hat{t}} S(\hat{t}-s) f(s, x(s)) d s \\
= & S\left(-k_{n}\right)\left[S\left(\hat{t}-a+k_{n}\right) x(a)+\int_{a}^{\hat{t}+k_{n}} S\left(\hat{t}-s+k_{n}\right) f(s, x(s)) d s\right] \\
& +\int_{\hat{t}+k_{n}}^{\hat{t}} S(\hat{t}-s) f(s, x(s)) d s \\
= & S\left(-k_{n}\right) x\left(\hat{t}+k_{n}\right)+\int_{\hat{t}+k_{n}}^{\hat{t}} S(\hat{t}-s) f(s, x(s)) d s .
\end{aligned}
$$

Hence

$$
\hat{x}-S\left(-k_{n}\right) x\left(\hat{t}+k_{n}\right)=\int_{\hat{t}+k_{n}}^{\hat{t}} S(\hat{t}-s) f(s, x(s)) d s, \quad n \in \mathbb{N} .
$$


Since

$\int_{\hat{t}+k_{n}}^{\hat{t}} S(\hat{t}-s) f(s, x(s)) d s=\int_{\hat{t}+k_{n}}^{\hat{t}} S(\hat{t}-s) f(\hat{t}, \hat{x}) d s+\int_{\hat{t}+k_{n}}^{\hat{t}} S(\hat{t}-s)[f(s, x(s))-f(\hat{t}, \hat{x})] d s$,

by means of the change of variables $\tau=\hat{t}-s$ introduced in the first integral on the right hand side, we have

$$
\begin{aligned}
\int_{\hat{t}+k_{n}}^{\hat{t}} S(\hat{t}-s) f(s, x(s)) d s & =\int_{0}^{-k_{n}} S(\tau) f(\hat{t}, \hat{x}) d \tau \\
& +\int_{\hat{t}+k_{n}}^{\hat{t}} S(\hat{t}-s)[f(s, x(s))-f(\hat{t}, \hat{x})] d s .
\end{aligned}
$$

Since both $f$ and $x$ are continuous functions in their respective domains, for every $\varepsilon>0$ there is $\sigma=\sigma(\varepsilon)>0$ such that

$$
\|f(t, x(t))-f(\hat{t}, \hat{x})\| \leq \varepsilon, \quad \text { for }|t-\hat{t}| \leq \sigma .
$$

Hence there is $\bar{n}=\bar{n}(\sigma)$ such that (5.6) is satisfied for $t \in\left[\hat{t}+k_{n}, \hat{t}\right]$ and $n \geq \bar{n}$. Consequently, since $\|S(t)\| \leq 1$ for $t \geq 0$ by (A), for $n \geq \bar{n}$ we have

$$
\begin{aligned}
\frac{1}{-k_{n}}\left\|\int_{\hat{t}+k_{n}}^{\hat{t}} S(\hat{t}-s)[f(s, x(s))-f(\hat{t}, \hat{x})] d s\right\| & \leq \frac{1}{-k_{n}} \int_{\hat{t}+k_{n}}^{\hat{t}}\|S(\hat{t}-s)\|\|f(s, x(s))-f(\hat{t}, \hat{x})\| d s \\
& \leq \frac{1}{-k_{n}} \varepsilon\left(-k_{n}\right)=\varepsilon .
\end{aligned}
$$

It shows that

$$
\frac{1}{-k_{n}} \int_{\hat{t}+k_{n}}^{\hat{t}} S(\hat{t}-s)[f(s, x(s))-f(\hat{t}, \hat{x})] d s \rightarrow 0, \quad \text { as } n \rightarrow \infty .
$$

Notice (see e.g. (2.1)) that

$$
\frac{1}{-k_{n}} \int_{0}^{-k_{n}} S(\tau) f(\hat{t}, \hat{x}) d \tau \rightarrow f(\hat{t}, \hat{x}), \quad \text { as } n \rightarrow \infty .
$$

Therefore, from conditions (5.4) and (5.5), we obtain

$$
\frac{S\left(-k_{n}\right) x\left(\hat{t}+k_{n}\right)-\hat{x}}{k_{n}} \rightarrow f(\hat{t}, \hat{x}) .
$$

We have then showed the existence of $\left\{\sigma_{n}\right\} \subset E$, depending on $\left\{k_{n}\right\}$, such that $\sigma_{n} \rightarrow 0$ as $n \rightarrow \infty$ and satisfying

$$
S\left(-k_{n}\right) x\left(\hat{t}+k_{n}\right)=\hat{x}+k_{n} f(\hat{t}, \hat{x})+k_{n} \sigma_{n} .
$$

Let $U \subset E$ be open with $\hat{x} \in U$ and $L>0$ be such that $\left.V\right|_{U}$ is $L$-Lipschitzian. Take $n$ large enough in such a way that both $\hat{x}+k_{n} f(\hat{t}, \hat{x})$ and $\hat{x}+k_{n} f(\hat{t}, \hat{x})+k_{n} \sigma_{n}$ belong to $U$ and $S\left(-k_{n}\right) x\left(\hat{t}+k_{n}\right) \in \bar{K}$ by (5.2). According to (V1) and (5.7) we obtain that

$$
0 \leq \frac{V\left(S\left(-k_{n}\right) x\left(\hat{t}+k_{n}\right)\right)}{k_{n}}=\frac{V\left(\hat{x}+k_{n} f(\hat{t}, \hat{x})+k_{n} \sigma_{n}\right)}{k_{n}}=\frac{V\left(\hat{x}+k_{n} f(\hat{t}, \hat{x})\right)}{k_{n}}+\Delta_{n},
$$


with

$$
\Delta_{n}:=\frac{V\left(\hat{x}+k_{n} f(\hat{t}, \hat{x})+k_{n} \sigma_{n}\right)-V\left(\hat{x}+k_{n} f(\hat{t}, \hat{x})\right)}{k_{n}} .
$$

By the L-Lipschitzianity in $U$ of $V$, we obtain that

$$
\left|\Delta_{n}\right| \leq L\left\|\sigma_{n}\right\| \rightarrow 0, \text { as } n \rightarrow \infty .
$$

Consequently,

$$
\begin{aligned}
\lim _{n \rightarrow \infty} \frac{V\left(\hat{x}+k_{n} f(\hat{t}, \hat{x})\right)}{k_{n}} & =\lim _{n \rightarrow \infty}\left[\frac{V\left(\hat{x}+k_{n} f(\hat{t}, \hat{x})\right)}{k_{n}}+\Delta_{n}\right] \\
& =\lim _{n \rightarrow \infty} \frac{V\left(S\left(-k_{n}\right) x\left(\hat{t}+k_{n}\right)\right)}{k_{n}} \geq 0
\end{aligned}
$$

in contradiction with (5.3). Hence $\hat{x} \in K$ and the proof is complete.

The case when the 0 -sublevel set of the bounding function is the ball centered in 0 and with radius $r$, i.e.

$$
V_{r}(x)=\frac{1}{2}\left(\|x\|^{2}-r^{2}\right), x \in E, r>0,
$$

frequently occurs in several applications (see e.g. Section 4).

Example 5.1. (i) Let $E$ be a Hilbert space with scalar product $\langle\cdot, \cdot\rangle$. In this case $V_{r} \in C^{1}(E)$ and $\dot{V}_{r}(x): E \rightarrow E$ is such that $\dot{V}_{r}(x)(y)=\langle x, y\rangle, x, y \in E, r>0$. Moreover $\left\|\dot{V}_{r}(x)\right\|=$ $\|x\|, x \in E$, and then $V_{r}$ is also locally Lipschitzian. Therefore, when the function $f$ satisfies

$$
\langle x, f(t, x)\rangle<0, \text { for } t \in(a, b] \text { and }\|x\|=r,
$$

then, by (5.1), $V_{r}$ is a locally Lipschitzian bounding function for (3.1) for all $r>0$.

(ii) Assume, now, that $E^{*}$ is a uniformly convex Banach space. Hence the function $V_{r}$ is Frechét differentiable on $E$ with

$$
\left\langle\dot{V}_{r}(x), y\right\rangle=\langle J(x), y\rangle, \quad \text { for } x, y \in E,
$$

where $J: E \rightarrow E^{*}$ is the single-valued duality map given by

$$
J(x)=x^{*} \in E^{*}:\left\|x^{*}\right\|=\|x\| \text { and }\left\langle x^{*}, x\right\rangle=\|x\|^{2}
$$

and $J$ is continuous (see e.g. [12]). Hence, if we further assume the existence of $r>0$ such that

$$
\langle J(x), f(t, x)\rangle<0, \text { for } t \in(a, b] \text { and }\|x\|=r,
$$

then $V_{r}$ is a locally Lipschitzian bounding function for equation (3.1).

In particular, let $E=L^{p}(\Omega)$ where $\Omega \subset \mathbb{R}^{n}$ is a bounded measurable subset of $\mathbb{R}^{n}, n \geq 1$ and $1<p<\infty$. Then $E$ is reflexive, $E^{*}=L^{p \prime}$, with $1 / p+1 / p^{\prime}=1$, is uniformly convex and (see [32, Example 1.4.4] and [16, Chapter I, Example 2.7])

$$
\langle J(x), y\rangle=\frac{1}{\|x\|^{p-2}} \int_{\Omega}|x(\xi)|^{p-2} x(\xi) y(\xi) d \xi, \quad x, y \in L^{p}(\Omega) .
$$


Again by (5.11), if the following condition

$$
\langle J(x), f(t, x)\rangle=\frac{1}{\|x\|^{p-2}} \int_{\Omega}|x(\xi)|^{p-2} x(\xi) f(t, x)(\xi) d \xi<0, \text { for } t \in(a, b] \text { and }\|x\|=r,
$$

is satisfied, then $V_{r}$ is a locally Lipschitzian bounding function for (3.1).

Example 5.2. In a uniformly convex Banach space E, consider the usual distance function $d(\cdot, r \bar{B}): E \rightarrow \mathbb{R}$ from the closed ball centered in 0 with radius $r>0$. From its definition,

$$
d(x, r \bar{B}):= \begin{cases}0 & \|x\| \leq r \\ \inf _{y \in r \bar{B}}\|x-y\|=\|x\|-r & \|x\|>r\end{cases}
$$

it is easy to show that it is a Lipschitzian function. If we further assume that

$$
\liminf _{h \rightarrow 0^{-}} \frac{d(x+h f(t, x), r \bar{B})}{h}<0, \quad \text { for } t \in(a, b],\|x\|=r,
$$

the function $d(\cdot, \bar{B})$ is a bounding function for equation (3.1).

\section{Acknowledgments}

The authors are members of the Gruppo Nazionale per l'Analisi Matematica, la Probabilità e le loro Applicazioni (GNAMPA) of the Istituto Nazionale di Alta Matematica (INdAM) and acknowledge financial support from this institution. The first author has been supported by the project Fondi Ricerca di Base 2016 Metodi topologici per equazioni differenziali in spazi astratti, Department of Mathematics and Computer Science, University of Perugia.

\section{References}

[1] J. Andres, L. Malaguti and V. Taddei, On boundary value problems in Banach spaces, Dynam. Systems Appl. 18 (2009), 275-302.

[2] R. A. Al-Omair and A. G. Ibrahim, Existence of mild solutions of a semilinear evolution differential inclusions with nonlocal conditions, Electron. J. Differential Equations 2009, No. $42,11 \mathrm{pp}$.

[3] I. Benedetti, N. V. Loi and V. Taddei, An approximation solvability method for nonlocal semilinear differential problems in Banach spaces, Discrete Cont. Dyn. Syst. 37,(2017), 2977-2998.

[4] I. Benedetti, V. Taddei and M. Väth, Evolution Problems with Nonlinear Nonlocal Boundary Conditions, J. Dynam. Differential Equations 25 (2013), 477-503.

[5] A. Boucherif and R. Precup, Semilinear evolution equations with nonlocal initial conditions, Dynam. Systems Appl. 16 (2007), 507-516.

[6] L. Byszewski, Theorems about the existence and uniqueness of solutions of a semilinear evolution nonlocal Cauchy problem, J. Math. Anal. Appl. 162 (1991), 494-505. 
[7] J. Chabrowski, On nonlocal problems for parabolic equations, Nagoya Math. J. 93 (1984), 109-131.

[8] T. Cardinali and P. Rubbioni, Aronszajn - Hukuhara type theorem for semilinear differential inclusions with nonlocal conditions, Electron. J. Qual. Theory Differ. Equ. 2015, No. 45, 12 pp.

[9] A. Ćwiszewski and P. Kokocki, Krasnoselskii type formula and translation along trajectories method for evolution equations, Discrete Contin. Dyn. Syst. 22 (2008), 605-628.

[10] K. Deng, Exponential decay of solutions of semilinear parabolic equations with nonlocal initial conditions, J. Math. Anal. Appl. 179 (1993), 630-637.

[11] E. Di Benedetto, U. Gianazza and V. Vespri, Harnack's inequality for degenerate and singular parabolic equations, Springer Monographs in Mathematics, Springer, New York, 2012 .

[12] J. Diestel, Geometry of Banach Spaces-Selected Topics, Lecture Notes in Mathematics, no. 485. Springer-Verlag, Berlin-New York, 1975.

[13] R. E. Gaines and J. L. Mawhin, Ordinary differential equations with nonlinear boundary conditions, J. Differential Equations 26 (1977), 200-222.

[14] R. E. Gaines and J. L. Mawhin, Coincidence Degree and Nonlinear Differential Equations. Lecture Notes in Mathematics, no. 568, Springer-Verlag, Berlin-New York, 1977.

[15] B. H. Gilding and R. Kersner, Travelling Waves in Nonlinear Diffusion-ConvectionReaction. Birkhäuser Verlag: Basel, 2004.

[16] S. Hu and N. Papageorgiou, Handbook of Multivalued Analysis Vol. I: Theory, Springer US, 1997.

[17] G. Infante and M. Maciejewski, Multiple positive solutions of parabolic systems with nonlinear, nonlocal initial conditions, J. Lond. Math. Soc. (2) 94 (2016), 859-882.

[18] D. Jackson, Existence and uniqueness of solutions to semilinear nonlocal parabolic equations, J. Math. Anal. Appl. 172 (1993), 256-265.

[19] M. Kamenskii, V. Obukhovskii and P. Zecca, Condensing Multivalued Maps and Semilinear Differential Inclusions in Banach Space, W. de Gruyter, Berlin, 2001.

[20] T. D. Ke, V. Obukhovskii, N. Wong and J. Yao, On semilinear integro-differential equations with nonlocal conditions in Banach spaces, Abstr. Appl. Anal. 2012, Art. ID 137576, 26 pp.

[21] P. Kokocki, Krasnosel'skii type formula and translation along trajectories method on the scale of fractional spaces, Commun. Pure Appl. Anal. 14 (2015), 2315-2334.

[22] M. A. Krasnosel'skii and P. P. Zabreiko, Geometrical Methods of Nonlinear Analysis. Izdat. Nauka, Moscow 1975; English translation, Springer-Verlag, Berlin, 1984. 
[23] J. Leray and J. Schauder, Topologie et équations fonctionnelles, Ann. Sci. École Norm. Sup. 51 (1934), 45-78.

[24] J. Liang, J. Liu and T. J. Xiao, Nonlocal Cauchy problems governed by compact operator families, Nonlinear Anal. 57 (2004), 183-189.

[25] A. Lunardi, Analytic semigroups and optimal regularity in parabolic problems, Progress in Nonlinear Differential Equations and their Applications, 16, Birkhuser Verlag, Basel, 1995.

[26] J. L. Mawhin and H. B. Thompson, Periodic or bounded solutions of Carathéodory systems of ordinary differential equations, J. Dynam. Differential Equations 15 (2003), $327-334$.

[27] J. C. Meyer and D. J. Needham, The Cauchy problem for non-Lipschitz semi-linear parabolic partial differential equations, London Mathematical Society Lecture Note Series, 419, Cambridge University Press, Cambridge, 2015.

[28] C. V. Pao, Reaction diffusion equations with nonlocal boundary and nonlocal initial conditions, J. Math. Anal. Appl. 195 (1995), 702-718.

[29] A. Pazy, Semigroups of Linear Operators and Applications to Partial Differential Equations, Springer-Verlag, Berlin, 1983.

[30] V. Taddei, Bound sets for Floquet boundary value problems: the nonsmooth case. Discrete Contin. Dynam. Systems 6 (2000), 459-473.

[31] A. Viorel, Nonlocal Cauchy problems close to an asymptotically stable equilibrium point, J. Math. Anal. Appl. 433 (2016), 1736-1742.

[32] I. I. Vrabie, Compactness Methods for Nonlinear Evolutions, 2nd ed., Longman House, Burn Mill, Harlow, 1990.

[33] I. I. Vrabie $C_{0}$-semigroups and applications, North-Holland Mathematics Studies, 191, North-Holland Publishing Co., Amsterdam, 2003.

[34] X. Xue, Nonlocal nonlinear differential equations with a measure of noncompactness in Banach spaces, Nonlinear Anal. 70 (2009), 2593-2601.

[35] A. Yagi, Abstract parabolic evolution equations and their applications, Springer Monographs in Mathematics. Springer-Verlag, Berlin, 2010.

[36] F. Zanolin, Bound sets, periodic solutions and flow-invariant for ordinary differential equations in $\mathbb{R}^{n}$ : some remarks, Rend. Istit. Mat. Univ. Trieste 19 (1987), 76-92.

[37] L. Zhu and G. Li, Existence results of semilinear differential equations with nonlocal initial conditions in Banach spaces, Nonlinear Anal. 74 (2011), 5133-5140.

[38] T. Zhu, C. Song and G. Li, Existence of mild solutions for abstract semilinear evolution equations in Banach spaces, Nonlinear Anal. 75 (2012), 177-181. 